\title{
Continuous glucose monitoring system (CGMS)
}

National Diabetes Information Clearinghouse (NDIC)

\section{Definitions}

Blood glucose level

Defined by National Diabetes Information Clearinghouse (NDIC)

\section{Source}

National Diabetes Information Clearinghouse (U.S.). (2009). The diabetes dictionary. [Bethesda, Md.]: U.S. Dept. of Health and Human Services, National Institutes of Health, National Institute of Diabetes and Digestive and Kidney Diseases, National Diabetes Information Clearinghouse.

A small sensor inserted below the skin that measures blood glucose levels approximately 12 times an hour. 